\title{
Parallel universes
}

\author{
Stephen B Hanauer
}

As specialists we seem to spend too much time talking amongst ourselves and not enough time talking to colleagues in other specialties. I believe we need to communicate more with those outside of our own fields so that we can consider alternative approaches to similar conceptual problems. Different specialties have a lot of problems in common, and the vertical (i.e. lack of) integration among specialties could be improved by more crosstalk. We can all learn from mature $B$ and $T$ lymphocytes, which interact more than we once appreciated!

In their commentary Clinical update: immunosuppression minimisation (Lancet [2007] 369: 1676-1678), Sayegh and Remuzzi consider transplantation. They point out that the main principle of immunosuppression in patients undergoing organ transplantation is combination treatment that targets distinct mechanisms of the immune response to control rejection, maintain long-term graft function, avoid infection and malignancy, and avoid drug toxicity. These authors also note, however, that studies of acute rejection are short term (6-12 months) and that "... little attention has been paid to late endpoints such as graft function, survival ... and novel endpoints and biomarkers of graft dysfunction."

Recent trials aimed at minimizing immunosuppression to prevent acute or chronic organ rejection have also focused on short-term endpoints that do not necessarily translate into long-term outcomes. Additional endpoints include steroid avoidance or withdrawal.

There are many similarities between the transplantation studies and studies of immunemediated inflammatory diseases. Most studies of IBD, for example, have been industrysupported, relatively short-term induction and (6-12 month) maintenance trials that have used various regimens (i.e. combination therapies)
What we

need now is crosstalk with our colleagues from ... other specialties ... so that we can modify the course of immunemediated inflammatory disorders

SB Hanauer is Editorin-Chief of Nature Clinical Practice Gastroenterology \& Hepatology.

\section{Competing interests}

The author declared he has no competing interests.

www.nature.com/clinicalpractice doi:10.1038/ncpgasthep0878 in the absence of predictable biomarkers or assays for immunosuppression and without consideration for long-term disease modification. In their article, Sayegh and Remuzzi call for clinical trials "...to be accompanied by extensive analyses of graft function, histology, assays to measure cellular and humoral immune responses, and assessment of the molecular phenotype of alloimmune response."

Combination therapy with corticosteroids, immunosuppressants and, most recently, biologic therapy targeting TNF are the current approaches to the treatment of moderate to severe IBD, and the goals of immunosuppression in chronic inflammatory diseases have been similar to those in transplantation. The shortterm and intermediate-term outcomes reflect neither the long-term outcomes (e.g. surgery) nor the risks (e.g. neoplasia). Prior recommendations for combined immunosuppression with biologic agents to avoid the development of immunogenicity have not translated into improved outcomes at 1 year and have been associated with an increased risk of lymphoma, particularly in young individuals who require lifelong treatment.

The IBD field is also moving to therapies that avoid or 'spare' corticosteroids and minimize immunosuppression to avoid short-term and long-term complications, such as infections and neoplasia. What we need now is crosstalk with our colleagues from the transplantation, rheumatologic, dermatologic and other specialties to identify appropriate functional analyses, histologic endpoints, assays to measure appropriate cellular and humoral immune responses, and molecular, genotypic, serologic, proteomic and metabolomic biomarkers to predict and measure the immune response and longterm outcomes so that we can modify the course of immune-mediated inflammatory disorders. 